\title{
V. Gardone-Riviera.
}

\author{
Ein Beitrag zur Klimatotherapie
} von

Ludwig Rohden.

Ich werde von mehreren Seiten aufgefordert, auclı der grösseren Collegenwelt Mittheilung zu machen von meiner Entdeckung einer Localität, welche sich nicht nur für die Wintermonate - für diese ist ja der Bedarf an Kurorten ziemlich gedeckt - , sondern - und dies ist eine grosse Seltenheit — ganz besonders für Herbst und Frühling qualificirt. Ein Ort, an dem die Uebergänge vom Sommer zum Winter und vom Winter zum Sommer fast unfühlbar, weil nahezu windstill von Statten gehen, ist, das wird ein jeder Kenner südlicher Kurorte zugestehen, eine Rarität und ein Desiderat. Und Gardone am Gardasee ist ein solcher Ort.

Vor $1 \frac{1}{2}$ Jahren erschienen zuerst in einzelnen Blättern bescheidene billige Annoncen, welche für das ganz unbekannte GardoneRiviera den Anspruch erhoben, so warm und so schön $\mathrm{zu}$ sein, wie die Riviera von Pegli, Argwöhnische sahen dam den Beinamen Riviera für ein Kennzeichen des Schwindels an. Selbst sehen und prüfen half mir dann im Herbst $\mathrm{zu}$ der Anschauung eines vortrefflichen deutschredenden, deutschreinlichen und deutschkochenden Hôtels, dessen Dörfchen im Gegensatze zu Gardone di sopra (G. am Berge) Gardone-Riviera (G. am Ufer) genannt wird. Ich war schon damals bezaubert von der frischen Vegetation, welche den Ort umgiebt und durch die Nachbarschaft des Sees nicht nur, sondern auch durch eine Menge kleiner Bäche unterhalten wird, welche in schattigen Thälern von den Bergen herabfliessen, aber ich dachte nicht daran, dass der Ort gerade etwas besonders Auszeichnendes haben könne.

$\mathrm{Da}$ bekamen zufällig im December einige deutsche Kurgäste zu Arco Verdruss mit ihrem Wirthe und kamen zu mir mit der Bitte, ihnen einen anderen in der Nähe gelegenen Ort anweisen zu wollen. Arco selbst sei ihnen durch jene Verdriesslichkeiten verleidet. Ich nannte ihnen Gardone. Zur Prüfung von Ort und Unterkunft wurde ein Kölner Herr mit schätzbaren Anlagen zu einem Lebemanne deputirt. Derselbe kam mit den günstigsten Nachrichten wieder, und die erste dauernde deutsche Colonie zog anderen Tages in Hôtel Gardone ein. Diese Colonie blieb die zweite Hälfte des Winters da, zog zahlreiche Nachzügler hinter sich her und gab mir Veranlassung zu einigen klimatologischen Wahrnehmungen, welche den grossen Werth Gardones darthun.

Eine Viertelstunde Gehens den Berg hinan und entlang liegt Cargnacco mit der Villa von Frau Emilie Wimmer, welche als Tochter eilles geschätzten österreichischen Arztes sich getrieben fühlte, ein meteorologisches Observatorium dort zu begründen. Möglicherweise ist die Temperatur dort im Ganzen etwas höher, als am See, so dass die Ergebnisse der in Cargnacco angelegten Beobachtungsstation höhere Wintertemperaturen und höhere Minima, als selbst Arco aufzuweisen hat - nicht unbedingt für Gardone am See zu verwenden sein werden, aber die Frage des Windschutzes von Gardone kann auch in Cargnacco entschieden werden, da dasselbe, weil höher, sogar noch exponirter liegt, als das erstere. Nun war im̉ März d. J. bei täglich drei, im Ganzen 93 Beobachtungen, die Luftbewegung $45 \mathrm{mal}$ eins, dreimal zwei, einmal drei und $44 \mathrm{mal}$ null, d. h. abso- 
lute Windstille, im April bei 90 Beobachtungen $20 \mathrm{mal}$ eins, zweimal zwei, viermal drei und 64 mal Windstille. Dies sind gewiss ganz besonders gute Ziffern für März und April. Ich habe selbst Gardone im vergangenen Frühling häufig besucht, habe ganz vorurtheilsfreie und wohlunterrichtete Kranke dort als Berichterstatter gehabt und kann versichern, dass alle einstimmig waren mir zu sagen, die Windstille, welche ich an meinen Besuchstagen beobachten konnte, sei keine Ausnahme, sondern die ständige Erscheinung des gesammten Frühlings. - Es giebt nur einen einzigen Wind, welcher die Bucht von Gardone stark bewegen macht, das ist der Südost, die Vinezza, wie sie die Eingeborenen nennen, weil sie von Venedig herbläst. Und dieser Südostwind ist, wie überall, so auch hier selten. Den See entlang blasen Nord-, Süd- und Südwestwind, auf ihm entsteht die sogleich zu besprechende Ora; aber Angesichts der eigenthümlichen Gestaltung des Sees und der Configuration seiner Ufer, welche die nördliche Hälfte zu einem Windrinnsal machen, kommt es bei diesen Nord- und Südwinden zwar oft zu heftiger Bewegung des Wassers und dann ist der Benacus in der That Fluctibus et fremitu marino assurgens, aber in Gardone sieht man diese Stürme nur in der Ferne auf dem See toben; Nachts muthmaasst man sie, wenn die Wellen stärker, als in der gewohnten weichen Weise ans Ufer schlagen.

Vom März bis October werden Riva und Arco täglich von der sogenannten Ora abgekühlt, einem Südwinde, welcher zwischen 10 und $11 \mathrm{Uhr}$ entsteht und bis gegen Abend anhält. Ueber diesen Wind ist schon wundersviel gesagt, aller Physik nach entsteht er auf folgende Weise: Die sonnendurchglühten Felder und Felsen des Sarcathales haben Vormittags bis gegen $11 \mathrm{Uhr}$ die darüberliegende Luft so stark erwärmt, dass sie, wie in einem Kamine in die Höhe steigend, die südwärts auf dem Wasser des Sees lagernde kühle Luftschicht berbeizieht. Lässt die Sonnenbestrahlung des Sarcathales Abends nach, so hört der Luftzug in demselben und somit auch das Naclrücken der Seeluft auf, es tritt Windstille eiu für die ganze Nacht bis zum folgenden Vormittage, falls die Sonne scheint. Keine Sonne, keine Ora! Die letztere kommt im Winter trotz Sonnenscheins deswegen nicht zu Stande, weil kalte lange Winternächte deı Erdboden so intensiv abkühlen, dass die Sonne am folgenden Tage bei ihrem winterlichen Tiefstande eine genügende Erwärmung des oft noch aufzuthauenden immer aber niedrig temperirten Bodens nicht zu leisten vermag. Im Herbst andererseits, wenn der See im Laufe des Sommers so warm geworden ist, dass er die über ihm lagernde Luft nicht mehr zur Genüge abzukühlen vermag, dann hört auch die Ora auf, weil der Gegensatz zu der warmen Luft des Sarcathales und seiner kahlen Bergwände nicht mehr gross genug ist, um einen Luftzug hervorzurufen, welcher ein Wind genannt werden muss. Ich gebe nach meinen persönlichen Erfahrungen des letzten Frühlings gern zu, dass dieser Wind in Riva und Arco sehr lästig zu werden vermag, theils durch Aufwirbeln von Staub, theils durch Beängstigung von Gemüthern, welche mehr oder weniger Ursache haben Windstille zu lieben und Wind zu scheuen. Diesen kann man dann nichts Zweckmässigeres rathen, als nach dem nahen Gardone sich zu flüchten, wo man weit draussen auf dem See das Wasser von der Ora kraus werden sieht, sonst aber Nichts von ihr bemerkt.

Unter diesen Windverhältnissen ist von Staub in der Luft absolut keine Rede, man vergisst einfach, dass es Staub giebt, wenn man ihn nicht durch geflissentliches Chausseelaufen auf die Stiefel ladet.

Das Hôtel in Gardone, vorläufig noch das einzige, mit demnächst ca. 80 Zimmern, missbraucht seine Alleinherrschaft in keiner Weise. Die Preise sind durchaus angemessen und im Verhältnisse zu anderen Etablissements der Art billig zu nennen. Unter einem wackeren Director aus Sachsen und seiner immer thätigen Frau, einer Schlesierin, lebt man so belaglich in italienischer Sonne und Flora, wie nur irgendwo auf deutschem Boden, für $6-7 \frac{1}{2}$ Mark per Tag. Sonstige Ausgaben fallen schon deshalb weg, weil keine Gelegenheit dazu vorhanden ist, man lebt still und gesetzt seinem Behagen, treibt sich in den Bergen herum und geht früh zu Bett.

Auch diese Einfachheit ist meiner Erfahrung nach ein grosser Vorzug Gardones.

Ich kann und mag zur Ehre der Menschheit nicht daran glauben, dass das Getïmmel grosser stark bevölkerter Kurorte, dass das Treiben von "Grand Hôtels" mit promptester Befriedigung aller Launen und Dummheiten reicher Leute das richtige Medium sei, in welchem sich Kranke und Erlolungsbedürftige aufhalten sollen. Ich bin im Gegentheile der Meinung, dass diese Art von Kurleben einer jeden auf Wiederherstellung ungünstig veränderter Körperverfassung bedacl-ten rationellen Methode direct widerspricht. Durch Unnatur kann man nicht zur Natur zurückkehren. Der Arzt muss deshalb nicht die odiöse und gewohnheitsmässige Fresserei einer $1 \frac{1}{2}$ stündigen
Table d'hôte für seine Kranken verlangen, sondern ein behagliches gemeinsames Essen von wenigen Gängen, wie die bessersituirte Familie es hat, muss das Erstrebenswerthe sein, nicht das fade geputzte Hinundwiederlaufen der sogenannten Kurpromenaden soll uuseren Kranken Bewegung machen, sondern das ungenirte Walldern in der Umgebung, das Steigen bergauf und bergab. Und nicht abendliche Bälle und Juchhe's in Gasluft, Staub und Anthropotoxin bringen unsere Phthisiker und Schwächlinge auf die Beine, sondern die Ruhe des Abends von entsprecliender körperlicher und leichter geistiger Arbeit ist voll Nöthell. Alles das liat man an kleinen Kurorten und in kleinen Hôtels, und ich stehe nicht an, es laut zu beklagen, dass manche zauberhaft schönen und auch gut gelegenen Orte sich durch Prachtbauten grossartiger Hôtels für Kranke einfach unbrauclıbar gemacht haben. Und dann, vergessen wir doch um Gotteswillen nicht, dass es dem Durchsclinitte unserer Kranken durchaus nicht gleichgiltig ist, ob er täglich 7 oder 20 Frcs. ausgiebt. Zum Gelingen einer Kur gehört auch das Behagen des Kranken und das Fernbleiben unangenehmer Eindrücke, das Behagen wird aber empfindlich gestört und zu den unangenehmsten Eindrücken gehört, wenn man sich bei jedem Essen und Trinken, bei jedem Glas Wein und bei jedem Bad, was man nehmen soll, sagen muss: um Gotteswillell, wie werde ich auskommen? Grands Hôtels haben grands prix, wenigstens an Kurorten, das steht fest, denn sonst können sie bei der kurzen Zeit ihrer Verwerthung nicht bestehen, und zu diesen grands prix gehört folgerichtig ein grand portemonnaie. Ich bin aber auch der Meinung, dass nur die Minderzahl der Reisenden darauf erpicht ist, Prunk und reiche Eleganz in den Hôtels zu finden. Den Meisten ist es sogar unangenehm, statt des ruhigen Comforts des eigenen Heims Prunkgemächer, wie die eines Fürstenschlosses, zu bewohnen, immer natürlich mit dem Bewusstsein, fürstlich dafür zahlen zu müssen. Auf kurzen Vergnügungsreisen tröstet man sich damit, dass man das Grab seiner Habe schön findet - „schön wars, aber verflucht kostspielig" - aber die Habe langt bei solchem Verfahren meist nicht auf die Zeit einer halbjährigen oder noch längeren Kur.

Aus therapeutischen, ethischen und finanziellen Gründen ziehe ich deswegen für Kurzwecke kleine Orte und kleine Hôtels vor. Wenı die letzteren glänzend reine Zimmer, ausgezeichnete Betten, gute Verpflegung, gute Closets, gute Badeeinrichtung und freundliche Leute haben, Alles für einen raisonnabeln Preis, dann sind sie so, wie sie sein sollen. Und so ist zu meiner grössten Freude das Hôtel Gardone und ich empfehle es mit dem Bewusstsein, dass mir Jeder, der dasselbe besucht, Dank dafür weiss.

Ich kann endlich niclit umhin, auch etwas ïber die landschaftliche Lage Gardones zu sagen.

Der Gardasee ist im Vergleiche zu den anderen oberitalienischen Seen, wie eine Schönheit im Battistkleidchen zu einer im Festgewande. $\mathrm{Da}$ ich die schöıe Natur der Ausstaffirung vorziehe, so ziehe ich auch den Gardasee vor und mit mir thut dies die Mehrzahl der Menschen, welche ich darüber befragt habe. Wie es unter diesen Umständen hat geschehen können, dass der Gardasee und seine Ufer so wenig bekannt und befahren sind, ist eine Frage, deren Beantwortung uns hier nichts angeht. Erinnert die nördliche Hälfte des Garda an die schönsten Theile des Königssees, so kann man für den südlichen Theil ohne jegliche Vebertreibung den Golf von Neapel zum Vergleiche heranziehen. Die Vegetation der Baien von Salo, Gardone und Maderno ist eine ganz südliche, die Citrone gedeilit mit ganz geringem Schutze gegen kalte Näclite zu einer weltberühmten Qualität, und „Lorbeerhaine", mit denen lächerlicherweise die Weltreklame der österreichischen Südbahn ihr für wahrhaft Kranke unbrauchbares Abbazia besonders herausstreicht, sind in Gestalt laubiger Wege viele Kilometer lang und reichlicher vorhanden, als den Landwirthen lieb ist. Musa Enseti und eine schöne Auzahl kräftiger Palmen steht ungedeckt oder kaum gedeckt im Freiell.

Die Reiserouten sind folgende: Durch den Gotthard fährt man über Mailand bis Desenzano, wo man Nachmittags Anschluss mit dem Dampfer hat, über den Brenner fährt man via Verona nach Desenzano oder via Mori nach Riva, wo man Morgens früh zu Schiffe geht. Beide Eisenbahnfahrten haben durchgehende Wagen bis Mailand resp. Verona. Eine sehr amüsante Sekundärbahn verbindet Brescia mit dem $1 / 2$ Stunde von G. entfernten Salo, bald auch mit Gardone selbst.

Aerztlicher Beistand wird von dem Schreiber dieses, welcher regelmässig von Arco herüberkommt und von Herrn Dr. Dus e, einem respectablen italienischen Collegen in Salo, geleistet. Für den Fall, dass man mich aus Arco nach modernsten politischen Principien entfernt, werde ich von October bis Mai in Gardone bleiben. Als nicht unerfahrener Klimatologus prophezeihe ich diesem Orte eine grosse Zukunft. Denn er ist klimatisch ganz vorzüglich, in mancher 
Beziehung einzig dastehend, er ist leicht zu erreichen, er ist billig und er wird von vernünftigen Principien regiert.

Und das sind 5 Eigenschaften, welche man selten vereint findet. 